\title{
Mujer joven con hepatocarcinoma fibrolamelar metastásico; citorreducción y HIPEC. A propósito de un caso
}

\author{
Jesús Morales Maza ${ }^{1}$, Óscar Santes ${ }^{1}$, Uriel Clemente-Gutiérrez ${ }^{1}$ y Rubén Cortés ${ }^{2}$
}

Young woman with metastatic fibrolamellar hepatocarcinoma; cytoreductive surgery and HIPEC. A case report

\begin{abstract}
Aim: To report a clinical case of metastatic fibrolamellar hepatocarcinoma and its multidisciplinary management. Case report: 24 year-old patient with abdominal pain, bloating and fever. A computed tomography of the abdomen was performed; an irregular hepatic tumor was found. A laparotomy was performed with evidence of multiple implants in the abdominal cavity and the histopathology report was metastatic fibrolamellar hepatocarcinoma. It was decided to perform cytoreductive surgery plus HIPEC. The patient's survival was 11 months. Discussion: Fibrolamellar hepatocarcinoma is a rare tumor. There is still no consensus on the treatment of choice in patients with metastases with good functionality status. Current management is based on systemic chemotherapy and surgical resection in localized cases. In the case of our patient, cytoreductive surgery plus HIPEC was performed with the intention of improving survival. More evidence is needed to define this strategy as standard treatment.
\end{abstract}

Key words: fibrolamellar hepatocarcinoma; cytoreductive surgery; intraperitoneal hyperthermic chemotherapy.

\section{Resumen}

Objetivo: Reportar un caso clínico de hepatocarcinoma fibrolamelar metastásico y su manejo multidisciplinario. Caso clínico: Paciente de 24 años de edad con dolor abdominal, distensión abdominal y fiebre. Se le realizó tomografía computarizada de abdomen donde se encontró tumoración hepática irregular. Se realizó laparotomía con evidencia de múltiples implantes en cavidad abdominal y se diagnosticó mediante estudio histopatológico hepatocarcinoma fibrolamelar metastásico. Se decidió realizar citorreducción más quimioterapia hipertérmica intraperitoneal (HIPEC). La sobrevida de la paciente fue de 11 meses. Discusión: El hepatocarcinoma fibrolamelar es un tumor raro. Aún no hay consenso sobre el mejor tratamiento en pacientes con metástasis que tengan buena funcionalidad. El manejo actual se basa en la quimioterapia sistémica y la resección quirúrgica en casos localizados. En el caso de nuestra paciente, la cirugía citorreductora más HIPEC se realizó con la intención de mejorar la supervivencia. Se necesita más evidencia para definir esta estrategia como tratamiento estándar.

Palabras clave: hepatocarcinoma fibrolamelar; citorreducción; quimioterapia hipertérmica intraperitoneal.

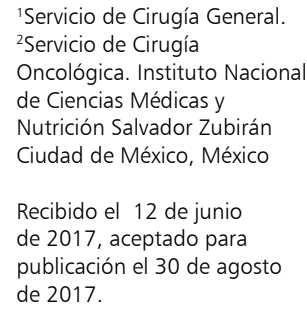

Correspondencia a: Dr. Jesus Morales M. medcardiouv@hotmail.com

\section{Introducción}

El hepatocarcinoma fibrolamelar se describió iniclamente en 1956 y se ha considerado como una variante rara del carcinoma hepatocelular con diferentes características clínico-patológicas. Representa del $1 \%$ al $9 \%$ de las variantes del hepatocarcinoma ${ }^{1}$.

\section{Caso clínico}

Presentamos el caso de una mujer de 24 años. Como único antecedente quirúrgico tenía una apendicectomía hace 15 años. Inicialmente presentó dolor abdominal en el hipocondrio derecho y epigastrio, síntomas gastrointestinales como náusea, vómito, plenitud postprandial y fiebre. Su examen 


\section{CASO CLÍNICO}

físico sin alteraciones. Se realizó tomografía computarizada y resonancia magnética (Figura 1) con evidencia de imagen nodular heterogénea ocupando el lóbulo hepático derecho con refuerzo heterogéneo y mal definido en la fase de contraste intravenoso
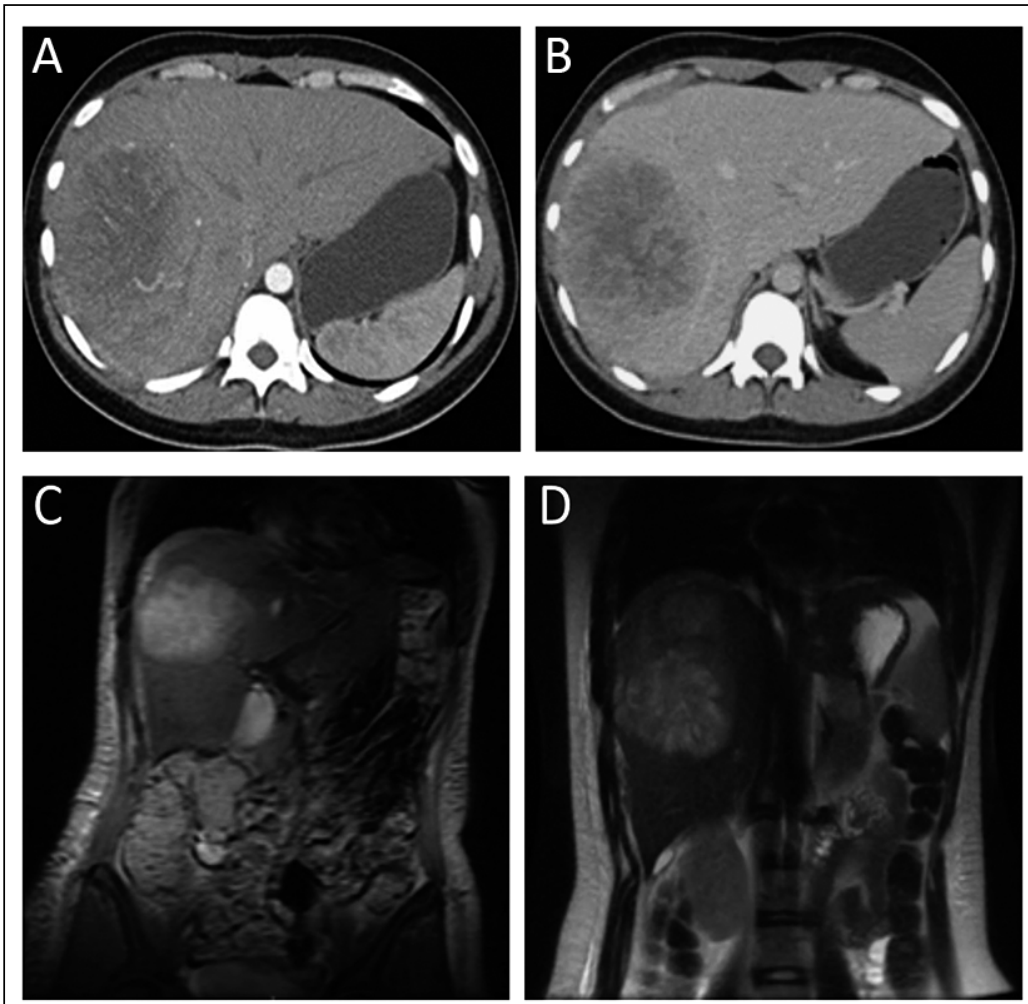

Figura 1. Lesión hepática en lóbulo hepático derecho compatible con hepatocarcinoma. A) Realce en fase arterial. B) Hipodensidad en fase portal. C) Resonancia magnética de la lesión en T1. D) Resonancia magnética de la lesión en T2. de $8,5 \times 9,5 \mathrm{~cm}$ y una calcificación gruesa de $3 \times 8$ $\mathrm{mm}$ en el interior. Sus pruebas de laboratorio eran normales.

Se decidió someter a la paciente a tratamiento quirúrgico. Se realizó laparotomía. Durante la cirugía se observaron múltiples implantes peritoneales con invasión de omento, diafragma derecho y útero. Además, se evidenciaron implantes múltiples en el mesenterio del sigmoides. Se envió una muestra de implante a biopsia rápida con reporte de hepatocarcinoma metastásico. Se calculó índice de carcinomatosis peritoneal con la escala de Sugarbaker ${ }^{2}$ el cual reveló una puntuación de 11 puntos. Se decidió cerrar la cavidad y no realizar la resección tumoral en ese momento.

El informe definitivo de patología informó hepatocarcinoma fibrolamelar metastásico.

Después de discutir el caso en un equipo multidisciplinario, debido a la edad de la paciente y su buena funcionalidad, se decidió realizar citoreducción más HIPEC (quimioterapia hipertérmica intraperitoneal). Se realizó hepatectomía derecha utilizando el aspirador quirúrgico ultrasónico de cavitrón (CUSA), resección de implantes mesentéricos, histerosalpingo-ooforectomía bilateral. El HIPEC se realizó usando mitomicina $\mathrm{C}$ durante 90 min a $41^{\circ} \mathrm{C}$ (Figura 2). Se dio de alta al día 10 posoperatorio.

Se realizó un seguimiento cada 2 meses después de la cirugía. La paciente logró una sobrevida de 11 meses y, finalmente, murió de obstrucción intestinal y sepsis abdominal.

Este tipo de tumores hepáticos se deben sospechar en pacientes jóvenes entre 10 y 30 años de edad sin antecedentes de cirrosis hepática ${ }^{3}$.

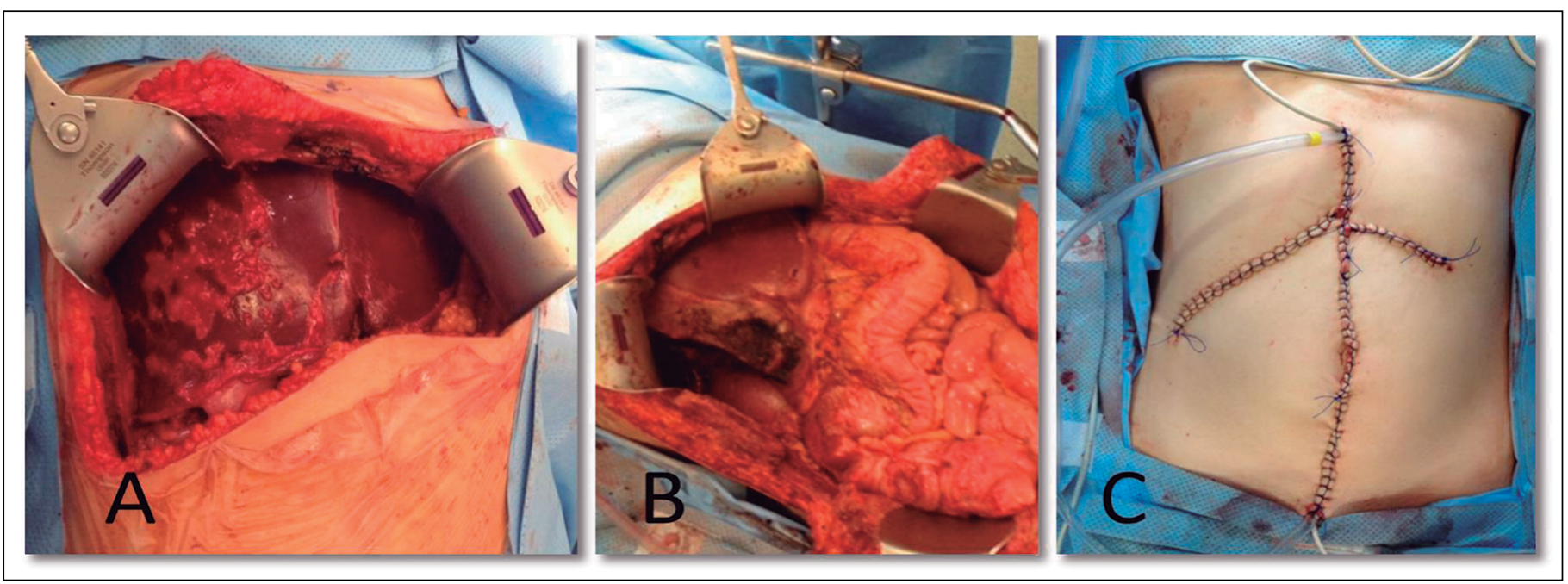

Figura 2. A) Invasión hepática tumoral. B) Hepatectomía derecha y citorreducción. C) HIPEC. 


\section{Discusión}

La etiología sigue siendo poco clara, aunque recientemente se ha asociado con factores de exposición ambiental y ocurre típicamente en pacientes con hígado $\mathrm{sano}^{4}$.

El enfoque diagnóstico inicial se realiza a través de estudios de imagen como ultrasonido y tomografía ${ }^{5}$.

El tratamiento descrito en la literatura del hepatocarcinoma fibrolamelar se ha basado en el mismo tratamiento de hepatocarcinoma hepatocelular. El tratamiento quirúrgico con resección hepática es un tratamiento seguro principalmente en hígados no cirróticos y es la única opción curativa, por tal motivo, actualmente es el tratamiento de elección en estos $\operatorname{casos}^{6,7}$.

Por otro lado, se ha descrito que el tratamiento quirúrgico de este tipo de tumores debe ser agresivo ya que se espera que estos pacientes tengan una larga supervivencia.

También se han logrado buenos resultados con el trasplante hepático en pacientes seleccionados con supervivencia similar a pacientes con diagnóstico de carcinoma hepatocelular ${ }^{8}$. La sobrevida de estos tumores es de aproximadamente $42 \%$ a $70 \%$ a los 5 años 9 . Algunos factores de mal pronóstico son la invasión de ganglios linfáticos y metástasis ${ }^{10}$.

\section{Conclusión}

En conclusión, el hepatocarcinoma fibrolamelar sigue siendo un tipo raro de tumor. En este momento no hay consenso sobre el mejor tratamiento en pacientes con metástasis que tengan buena funcionalidad. El manejo actual se basa en la quimioterapia sistémica y la resección quirúrgica en casos localizados. En el caso de nuestra paciente, la cirugía citorreductora más HIPEC se realizó con la intención de mejorar la supervivencia. Se necesita más evidencia para definir esta estrategia como tratamiento estándar.

\section{Responsabilidades éticas}

Protección de personas y animales. Los autores declaran que para esta investigación no se han realizado experimentos en seres humanos ni en animales.

Confidencialidad de los datos. Los autores declaran que han seguido los protocolos de su centro de trabajo sobre la publicación de datos de pacientes.

Derecho a la privacidad y consentimiento informado. Los autores declaran que en este artículo no aparecen datos de pacientes.

Conflictos de interés: No hay.

\section{Referencias}

1. Riggle KM, Turnham R, Scott JD, Yeung RS, Riehle KJ. Fibrolamellar Hepatocellular Carcinoma: Mechanistic Distinction From Adult Hepatocellular Carcinoma. Pediatr Blood Cancer. 2016;63:1163-7.

2. Sugarbaker PH. Cytoreductive surgery and hyperthermic intraperitoneal chemotherapy in the management of gastrointestinal cancers with peritoneal metastases: Progress toward a new standard of care. Cancer Treat Rev. 2016;48:42-9.

3. Lafaro KJ, Pawlik TM. Fibrolamellar hepatocellular carcinoma: current clinical perspectives. J Hepatocell Carcinoma. 2015;2:151-7.

4. Graham RP, Craig JR, Jin L,
Oliveira AM, Bergquist JR, Truty MJ, et al. Environmental exposures as a risk factor for fibrolamellar carcinoma. Mod Pathol. 2017;30:892-6.

5. Estrella Díez E, Álvarez Higueras FJ, Marín Zafra G, Bas Bernal Á, Garre Sánchez C, Egea Valenzuela J, et al. Fibrolamellar hepatocellular carcinoma: a rare entity diagnosed by abdominal ultrasound. Rev Esp Enferm Dig. 2016;108:494-5.

6. Faber W, Sharafi S, Stockmann M, Dennecke T, Bahra M, Klein F, et al Patient age and extent of liver resection influence outcome of liver resection for hepatocellular carcinoma in noncirrhotic liver. Hepatogastroenterology. 2014;61:1925-30.

7. Kassahun WT. Contemporary management of fibrolamellar hepatocellular carcinoma: diagnosis, treatment, outcome, prognostic factors, and recent developments. World J Surg Oncol. 2016;14:151.

8. Atienza LG, Berger J, Mei X, Shah MB, Daily MF, Grigorian A, et al. Liver transplantation for fibrolamellar hepatocellular carcinoma: A national perspective. J Surg Oncol. 2017;115:31923.

9. Lim II, Farber BA, LaQuaglia MP. Advances in fibrolamellar hepatocellular carcinoma: a review.Eur J Pediatr Surg. 2014;24:461-6.

10. Darcy DG, Malek MM, Kobos R, Klimstra DS, DeMatteo R, La Quaglia MP. Prognostic factors in fibrolamellar hepatocellular carcinoma in young people. J Pediatr Surg. 2015;50:153-6. 